\title{
Multi-detector computed tomography radiation doses in the follow-up of paediatric neurosurgery patients in KwaZulu-Natal: A dosimetric audit
}

\begin{abstract}
Authors:
Christopher T. Sikwila

Khatija Amod ${ }^{2}$

Brindley D. Cupido ${ }^{3}$

Ali Sabri ${ }^{4}$

Affiliations:

${ }^{1}$ Department of Radiology, Nelson R. Mandela School

of Medicine, University of

KwaZulu-Natal, South Africa

2Department of Radiology, Inkosi Albert Luthuli Central
\end{abstract} Hospital, South Africa

${ }^{3}$ Department of Radiology, Addington Hospital,

South Africa

${ }^{4}$ Department of Radiology, Cypress Health Region

Hospital, Canada

Correspondence to: Christopher Sikwila

Email:

tcsikwila@yahoo.co.uk

Postal address:

PO Box 30904, Lusaka 10101,

Zambia

Dates:

Received: 03 Nov. 2013

Accepted: 28 Nov. 2013

Published: 09 May 2014

How to cite this article: Sikwila CT, Amod K, Cupido, BD, Sabri A. Multi-detector computed tomography radiation doses in the follow-up of paediatric neurosurgery patients in KwaZulu-Natal: A dosimetric audit. S Afr J Rad. 2014;18(1); Art. \#588, 4 pages. http:// dx.doi.org/10.4102/sajr. v18i1.588

\section{Copyright:}

(C) 2014. The Authors.

Licensee: AOSIS

OpenJournals. This work

is licensed under the

Creative Commons

Attribution License.

\section{Read online:}

Background: Multi-detector computed tomography (MDCT) is the preferred modality for follow-up of paediatric neurosurgery patients. Serial imaging, however, has the disadvantage of an ionising radiation burden, which may be mitigated using the 'as low as reasonably achievable' (ALARA) principle.

Objectives: The primary objectives were to determine the radiation dose exposure in paediatric patients subjected to MDCT imaging following neurosurgery and to compare these values with references in current literature. Our secondary objective was to assess the relationship between radiation dose and clinical scenario.

Method: Retrospective descriptive data were collected from all paediatric postsurgical patients $(n=169)$ between the ages of 0 and 12 years who had their first followed-up scan in the year 2010 and were followed up for six months or less. Dose-length product (DLP) and current-time product were collected from the picture archiving and communication system. Demographic data including radiology reports were collected from the hospital information system. The effective doses (ED) were calculated from the corresponding DLP using age-adjusted conversion factors. For purposes of comparison with other studies, median dosimetric values were calculated and the children were grouped into three age ranges, namely younger than 3 years, 3-7 years and 8-12 years old.

Results: The highest median radiation doses were noted in patients being followed-up for intracranial abscesses $(1183 \mathrm{mGy} \mathrm{cm})$ in the 8-12 year age group, most of whom were female. The lowest radiation doses were for intracranial shunt follow-ups (447 $\mathrm{mGy} \mathrm{cm}$ ). Median values for DLP, ED and current-time product (mAs) were comparable to reference doses in all three age groups. However, our study showed a much broader distribution of values with higher upper limits relative to reference values. Indications for follow-up included shunts $(n=110 ; 65 \%)$, intracranial abscess $(n=31 ; 18 \%)$, subdural haematoma $(n=13 ; 8 \%)$ and tumour $(n=6 ; 4 \%)$. Head trauma only accounted for $5 \%$ of the cases.

Conclusion: The median radiation doses measured were comparable to values in literature and therefore deemed acceptable. The wider dose distributions of all three dosimetric parameters (DLP, ED and mAs) were attributed to inappropriate use of scan length and reference effective mAs. Adherence to recommended scan length protocols should be encouraged. Evaluation of the current use of reference effective mAs is needed and will require a separate study to determine the smallest value that can be used without compromising image quality. Further dose reductions could be achieved by omission of unenhanced scans in the follow-up of intracranial abscesses. It is recommended that diagnostic reference levels specific to South African clinical scenarios be developed to make local dosimetric audits more relevant.

\section{Introduction}

Children treated for complex or chronic neurological disease such as hydrocephalus, tumours and intracranial abscess often undergo serial imaging studies with multi-detector computed tomography (MDCT). The associated ionising radiation has raised concern as it is the dominant contributor to radiation dose from medical x-rays. The Department of Neurosurgery at Inkosi Albert Luthuli Central Hospital (IALCH) has a heavy case load as it serves the entire province of KwaZulu-Natal. Current protocols permit neurosurgeons to order computed tomography (CT) scans without prior consultation with a radiologist in order to increase efficiency in light of human resource constraints. As a result, an increase in the number of serial scans in the followup of paediatric patients was noted, raising concerns over excessive radiation burden due to the long-term increased risk of developing malignancies such as leukaemia, thyroid carcinomas, breast cancer as well as damage to the lens of the eye. Children and women in particular are more radiosensitive because of a higher rate of cell division in the former and radiosensitive 
organs being anatomically closer to the primary beam in the latter. However, there are no 'normal limits' with respect to radiation exposure to children in diagnostic imaging. Radiologists and medical physicists depend on the 'as low as reasonably achievable' (ALARA) principle and diagnostic reference levels (DRLs) ${ }^{1,2}$ to minimise the radiation exposure. South Africa has not yet developed DRLs with which we could compare our data; consequently comparisons were made with European DRLs/data sets predominantly derived from head trauma centres. The study was aimed at quantifying the radiation burden and comparing it to accepted values in literature. The outcomes would be beneficial in deciding whether to alter current paediatric protocols and establish benchmarks for future dosimetric audits.

\section{Methods and materials}

Ethical approval was obtained from the Biomedical Research and Ethics Committee of the University of KwaZuluNatal. Retrospective descriptive data was collected from all paediatric postsurgical patients $(n=169)$ between the ages of 0 and 12 years who had their first follow-up scan in the year 2010 and were followed up for six months or less. The 16-slice and 128-slice CT scanners used were calibrated by independent inspection bodies and dose parameters were checked and verified for accuracy to within $15 \%$ of the baseline values.

Dose-length product (DLP) and current-time product are parameters recorded by the MDCT machines used at the time of the scan and were thus collected from the picture archiving and communication system. Demographic data including radiology reports were collected from the hospital information system. The effective doses (ED) were calculated from the corresponding DLP using age-adjusted conversion factors (see Table 1) and the following equation:

$\mathrm{ED}=\mathrm{E}_{\mathrm{DLP}} \times \mathrm{DLP}(\mathrm{mSv})$, where:

$\mathrm{DLP}(\mathrm{mGy} \mathrm{cm})$ is the dose-length product, and

$\mathrm{E}_{\mathrm{DLP}}$ is the age-specific normalised ED per DLP

$\left(\mathrm{mSv} \mathrm{mGy}^{-1} \mathrm{~cm}^{-1}\right)$.

[Eqn 1]

For purposes of comparison with other studies, median dosimetric values were calculated and the children were grouped into three age ranges, namely younger than 3 years, 3-7 years and 8-12 years old.

\section{Results}

The highest median radiation doses were noted in patients being followed-up for intracranial abscesses (1183 mGy $\mathrm{cm}$ ) in the 8-12 year age group, most of whom were female. The lowest radiation doses were noted in intracranial shunt follow-ups (447 mGy cm). Median values for DLP, ED and current-time product (mAs) were comparable to reference doses in all three age groups. However, our study showed a much broader distribution of values with higher upper limits relative to reference values. Seventy-nine $(47 \%)$ of those scanned were male and $90(53 \%)$ were female. Median age was 2 years (range 1-12 years). The age distribution between the three age groups $<3,3-7$ and $8-12$ years were $98(58 \%)$, $34(20 \%)$ and $37(22 \%)$ respectively. The female distribution in the study was $51(57 \%), 17(19 \%)$ and $22(24 \%)$ and male distribution 47 (59\%), $17(22 \%)$ and $15(19 \%)$ children in the $<3,3-7$ and $8-12$ year age groups respectively. Indications for follow-up included: shunt $(n=110 ; 65 \%)$, intracranial abscess $(n=31 ; 18 \%)$, subdural haematoma or $\mathrm{SDH}(n=13$; $8 \%)$, trauma $(n=8 ; 5 \%)$, tumour $(n=6 ; 4 \%)$ and other $(n=1$; $<1 \%$ ) (see Figure 1).

No interval change between scans was reported by the radiologist in $65(59 \%)$ of shunt, $13(42 \%)$ of intracranial abscess, $7(50 \%)$ of SDH, $3(31 \%)$ of trauma and $4(67 \%)$ of tumour follow-ups. With respect to radiation burden attracted by an examination indication, our study showed the median DLPs (mGy cm) to be 447 (shunts), 1183 (intracranial abscess), 719 (SDH), 681 (trauma) and 591 (tumour). Sixty-eight percent of two-phase scans (i.e. with and without contrast material) were done in the 8-12 year age group, $22 \%$ in the $<3$ year age group and $47 \%$ in the $3-7$ year age group.

The median DLP (with range indicated in parenthesis) for the $<3,3-7$ and $8-12$ year age groups were 463 (251-1461), 615 (360-1268) and 1134 (393-3111) mGy cm respectively. Associated median effective doses were 3.1 (1.7-9.8), 2.5 (1.4-5.1) and $3.6(1.3-10.0) \mathrm{mSv}$ respectively. The median current-time product for the same age groups were 122 (89-350), 137 (107-300) and 191 (123-360) mAs respectively.

\section{Discussion}

Children treated for complex or chronic neurological disease often undergo serial imaging studies with MDCT. As mentioned, the associated ionising radiation has raised concern as it is the dominant contributor to radiation dose from medical x-rays. In this study, $141(83 \%)$ of the cases

TABLE 1: $E_{D L P}$ values used in this study. ${ }^{3}$

\begin{tabular}{ll}
\hline Age group (years) & $\mathrm{E}_{\mathrm{DLP}}$ in head region \\
\hline $1-3$ & 0.0067 \\
$3-7$ & 0.004 \\
$8-12$ & 0.0032 \\
\hline
\end{tabular}

$E_{D L P}$, age-specific normalised ED (effective dose) per DLP (dose-length product).

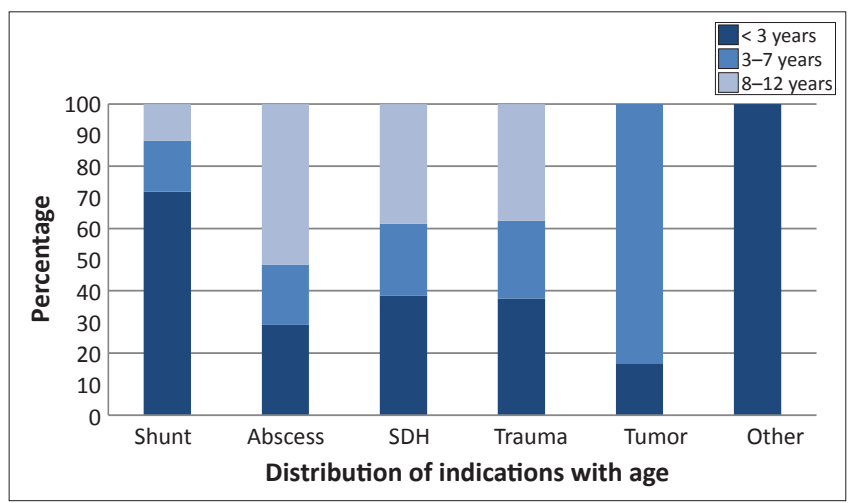

SDH, subdural haematoma.

FIGURE 1: Distribution of scan indications with age group. 
were due to shunt or intracranial abscess follow-up - the latter carrying a large radiation burden due to the two-phase scans done in accordance with neurosurgery protocols. Udayasankar et al. ${ }^{4}$ recommended the use of $80 \mathrm{kV} / 80 \mathrm{mAs}$ for shunt follow-up and $80 \mathrm{kV} / 90-140 \mathrm{mAs}$ for follow-up of abscesses and tumours as an alternative low-dose protocol. Rybka, Staniszewska and Biegański ${ }^{5}$ showed that as much as $70 \%$ reduction in patient doses were achieved without compromising image quality and that a low-dose protocol was feasible.

Notwithstanding the use of the reference effective mAs function on CT scanners, the upper limit of the mAs in children younger than 7 years was higher than those noted by Pages, Buls and Osteaux ${ }^{6}$ despite the median values being comparable (see Table 2). We attributed the finding to the inappropriately high reference effective mAs used on the 128-slice machine (see Table 3). Given that the mAs is directly proportional to the radiation dose, $\mathrm{Yu}$, Bruesewitz, Thomas,

TABLE 2: Dose and current-time product distribution with age.

\begin{tabular}{|c|c|c|c|}
\hline \multirow[t]{2}{*}{ Median values } & \multicolumn{3}{|c|}{ Age } \\
\hline & $<3$ years & $3-7$ years & $8-12$ years \\
\hline \multicolumn{4}{|l|}{ Dose-length product (mGy cm) } \\
\hline $\mathrm{IALCH}$ & $\begin{array}{c}463 \\
(251-1461)\end{array}$ & $\begin{array}{c}615 \\
(360-1268)\end{array}$ & $\begin{array}{c}1134 \\
(393-3111)\end{array}$ \\
\hline UK 2003 & 270 & 470 & 620 \\
\hline $\begin{array}{l}\text { Buls, Bosman, Mommeart, } \\
\text { Malchair,Clapuyt and Everarts } \dagger\end{array}$ & $216-352$ & 468 & 442 \\
\hline Freiberg, Almen, Einarsson et al. $\dagger$ & $394-472$ & $558-614$ & 580 \\
\hline Pages, Buls and Osteaux'ं & $117-248$ & $108-477$ & $228-954$ \\
\hline \multicolumn{4}{|l|}{ Effective dose (mSv) } \\
\hline IALCH & $\begin{array}{c}3.1 \\
(1.7-9.8)\end{array}$ & $\begin{array}{c}2.5 \\
(1.4-5.1)\end{array}$ & $\begin{array}{c}3.6 \\
(1.3-10.0)\end{array}$ \\
\hline Freiberg, Almen, Einarsson et al. $\dagger$ & $3.3-5.5$ & 2.3 & 2.3 \\
\hline Pages Buls and Osteaux † & $0.31-1.46$ & $0.41-1.57$ & $0.56-2.26$ \\
\hline \multicolumn{4}{|l|}{ Effective current-time product (mAs) } \\
\hline IALCH & $\begin{array}{c}122 \\
(89-350)\end{array}$ & $\begin{array}{c}137 \\
(107-300)\end{array}$ & $\begin{array}{c}191 \\
(123-360)\end{array}$ \\
\hline Pages Buls and Osteaux $\dagger$ & $75-225$ & $75-225$ & $225-300$ \\
\hline
\end{tabular}

$\dagger$, Values are approximations due to the overlapping age ranges of comparative studies. Ranges denoted in brackets.

UK 2003 DRL, Mean values: Shrimpton, Hillier, Lewis and Dunn ${ }^{10}$; Effective dose, ED (mSv) $E_{D L P} \times D L P$, where $E_{D L P}=$ age-specific normalised $E D$ per DLP $\left(m S v m G y^{-1} \mathrm{~cm}^{-1}\right)$ and DLP $=$ dose length product $(\mathrm{mGy} \mathrm{cm})$.

DRL, diagnostic reference levels.

TABLE 3: Scan parameters.

\begin{tabular}{lccc}
\hline Parameter & $\begin{array}{c}\text { 16-Slice } \\
\text { MDCT }\end{array}$ & $\begin{array}{c}\text { 128-Slice } \\
\text { MDCT }\end{array}$ & $\begin{array}{c}\text { Buls, Bosmans, } \\
\text { Hommaert, } \\
\text { Malchair, Clapuyt } \\
\text { and Everarts }\end{array}$ \\
\hline Tube potential (kV) & 120 & 120 & 120 \\
Pitch & 0.55 & 0.8 & $0.63-0.65$ \\
Rotation (sec) & 0.75 & 1.0 & - \\
FOV (mm) & 180 & 180 & - \\
Slice thickness (mm) & 10 & 5 & - \\
Reference effective mAs & 150 & 350 & - \\
Scan length (cm): 0-1 years & - & - & $8-11 \dagger$ \\
Scan length $(\mathrm{cm}): 1-5$ years & - & - & $11-13 \dagger$ \\
Scan length $(\mathrm{cm}): 5-15$ years & - & - & $(280-385 \mathrm{mG} \mathrm{cm})$ \\
& & & $13 \dagger$ \\
\end{tabular}

MDCT, multi-detector computed tomography.

(Approximate calculated DLP [dose-length product]).

$\dagger$, Shrimpton.
Fletcher, Kofler and McCollough 7 noted a 40\% - 50\% reduction in radiation dose with correct use of such AEC (automatic exposure control) systems. Radiologists have to be aware that these systems control radiation exposure relative to the required image quality (as determined by the reference effective $\mathrm{mAs}$ ), rather than decrease radiation dose directly. ${ }^{8,9}$

The median values for DLP for the $<3$ and 3-7 year age groups in our study were comparable to those obtained by Buls, Bosmans, Mommaert, Malchair, Clapuyt and Everarts ${ }^{8}$ and Freiberg, Almen, Einarsson et al. ${ }^{11}$, most likely due to the fact that their studies, like ours, included MDCT (rather than single-slice) machines as well as two-phase scans. The median DLP for the 8-12 year age group, however, was higher by a factor of 1.7-2.0. It is in this age group that we found the highest indication for intracranial abscess followup and consequently the most two-phase scans and greatest radiation dose burden (see Figures 1-3).

Furthermore, given that $42 \%$ of the scan follow-ups for intracranial abscess had no interval change identified on follow-up and that $22(59 \%)$ of the children in this age group were female, targeted application of a low-dose protocol and stringent assessment of the risk-benefit ratio for each CT request would have the greatest impact on dose reduction. The DLP distribution in all three age groups were much greater than DRLs of similar studies in literature (see Table 2), attributable to the high variation in scan lengths used. Buls, Bosmans, Mommaert, Malchair, Clapuyt and Everarts $^{8}$, using tube potential and pitch similar to that in this study, had upper limit values for DLP which were smaller

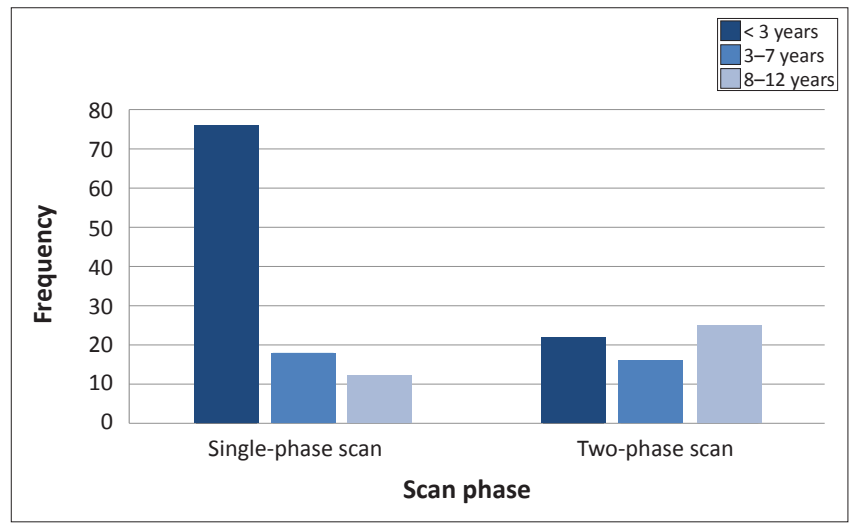

FIGURE 2: Frequency of single and two-phase scans.

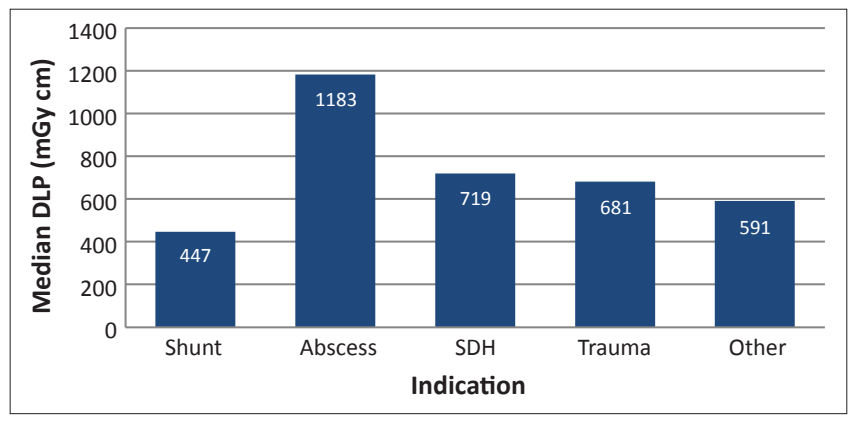

DLP, dose-length product; SDH, subdural haematoma.

FIGURE 3: Radiation burden of different clinical scenarios. 
by a factor of 2.7-7 for comparative age ranges. Calculations based on figures supplied by Buls, Bosmans, Mommaert, Malchair, Clapuyt and Everarts ${ }^{8}$ for the radiation doses expected given similar technique parameters to our study (see Table 3) suggest the scan lengths used at IALCH were greater by a factor of approximately $2-5$ compared to those recommended by Shrimpton ${ }^{3}$.

ED calculations from DLP values provide for a simple method for radiologists without access to medical physicists to obtain valuable information regarding the radiation exposure associated with their scanners or protocols. ${ }^{12}$ The median ED in our study were comparable to those of Friberg, Almen, Einarsson et al.'s study for the $<3$ and 3-7 year age groups; however, the 8-12 year age group had a higher dose by a factor of 1.6 due mainly to the large number of twophase scans. The higher median ED noted in the $<3$ age group compared to the 3-7 age group despite a lower DLP was also noted in the Freiberg, Almen, Einarsson et al. study, in which they attributed it to scan length discrepancies. We believe, however, that this difference is more a reflection of the higher conversion coefficients used to calculate ED in this age group.

Study limitations include non-separation of the neonatal group from the $<3$ year age grouping. The calculated effective doses were all done using the 1-3 age group conversion coefficient of 0.0063 instead of the higher 0.011 of the $<1$ year age group. Doses for the highly radiosensitive neonate were thus not captured and the calculated ED for the $<3$ year age group in our study was therefore an underestimation of the true ED. The study represented a radiation burden from CT head scans only and is not representative of the total radiation exposure during the relevant six-month period, that is, from CT scans of other body parts or daily chest x-rays in ICU. South Africa has not developed DRLs with which we could compare our data; consequently comparisons were made with European data sets which were predominantly derived from head trauma centres.

\section{Conclusion}

Our results show that radical changes to the existing paediatric protocols are not necessary given that the average DLP, ED and mAs values used were within acceptable limits compared to current literature. The marked variations in dose distribution were of concern, however, and were attributed to the inappropriate selection of scan length and operator-dependent reference effective mAs. Corrective strategies should include strict adherence to recommended scan length protocols, application of the ALARA principle and evaluation of the current use of reference effective $\mathrm{mAs}$ (which will require a separate study to determine the smallest value that can be used without compromising image quality). Further dose reductions with respect to intracranial abscess follow-up may be achieved by omitting the pre-contrast scan. Finally, future dosimetric audits would be greatly improved if national DRLs were developed which would reflect more common clinical scenarios such as intracranial sepsis.

\section{Acknowledgements}

We gratefully acknowledge Dr William Rae (Associate Professor, Medical Physics, University of the Free State) for his invaluable insight into the development of the article.

\section{Competing interests}

The authors declare that they have no financial or personal relationship(s) that may have inappropriately influenced them in writing this article.

\section{Authors' contributions}

C.T.S. (University of KwaZulu-Natal) was the principal author, whilst K.A. (Inkosi Albert Luthuli Central Hospital), B.D.C. (Addington Hospital) and A.S. (Cypress Health Region Hospital) made conceptual contributions.

\section{References}

1. ICRP. Recommendations of the International Commission on Radiological Protection. ICRP Publication 103. Ann ICRP. 2007;37(2-4).

2. ICRP. Radiological Protection and Safety in Medicine. ICRP Publication 73. Ann ICRP 1996;26(2), 1-47. http://dx.doi.org/10.1016/S0146-6453(00)89195-2

3. Shrimpton PC. Assessment of patient dose in CT. NRPB- PE/1/2004. Chilton: National Radiological Protection Board; 2004.

4. Udayasankar UK, Braithwaite $\mathrm{K}$, Arvaniti $\mathrm{M}$, et al. Low-dose non-enhanced head CT protocol for follow-up evaluation of children with ventriculoperitoneal shunt: Reduction of radiation and effect on image quality. Am J Neuroradio. 2007;29:802-806. http://dx.doi.org/10.3174/ajnr.A0923

5. Rybka K, Staniszewska AM, Biegański T. Low-dose protocol for head CT in monitoring hydrocephalus in children. Med Sci Monit. 2007;13(suppl 1):147-151. PMID: 17507900

6. Pages J, Buls N, Osteaux M. CT doses in children: A multicenter study. Br J Radiol. 2003;76:803-811. http://dx.doi.org/10.1259/bjr/92706933

7. Yu L, Bruesewitz MR, Thomas KB, Fletcher JG, Kofler JM, McCollough CH. Optimal tube potential for radiation dose reduction in pediatric $\mathrm{CT}$ : Principles, clinical implementations, and pitfalls. Radiographics. 2011;31:835-848. http://dx.doi. org/10.1148/rg.313105079

8. Buls N, Bosmans H, Mommaert C, Malchair F, Clapuyt P, Everarts P. CT pediatric doses in Belgium: A multi-centre study. Brussels: Brussels Free Universities; 2010.

9. Lee $\mathrm{CH}$, Goo JM, Lee $\mathrm{HJ}$, et al. Radiation dose modulation techniques in the multidetector CT era: From basics to practice. Radiographics 2008;28:1451-1459. http://dx.doi.org/10.1148/rg.285075075

10. Shrimpton PC, Hillier MC, Lewis MA, Dunn M. Dose from computed tomography (CT) examination in the UK. Br J Radiol. 2006;79:968-980. http://dx. doi. org/10.1259/bjr/93277434

11. Friberg EG, Almen A, Einarsson $\mathrm{G}$, et al. Doses from pediatric $\mathrm{CT}$ examinations and level of optimization of the scan protocols in the Nordic countries. Proceedings of the 15th Nordic Society for Radiation Protection (NSFS) conference; 2008 May 26-30; Ålesund, Norway. NSFS; 2008. p. 41-50.

12. Thomas KE, Wang B. Age-specific effective dose for pediatric MSCT examinations at a large children's hospital using DLP conversion coefficients: A simple estimation method. Pediatr Radiol. 2008;38:645-656. http://dx.doi.org/10.1007/ s00247-008-0794-0 